\title{
Band Gaps in Metamaterial Plates: Asymptotic Homogenization and Bloch-Floquet Approaches
}

\author{
David Faraci $^{1}$ (D) Claudia Comi ${ }^{1}$ (D) Jean-Jacques Marigo ${ }^{2}$
}

Received: 10 June 2021 / Accepted: 16 January 2022 / Published online: 11 February 2022

(c) The Author(s) 2022

\begin{abstract}
In this work, we study the transversal vibration of thin periodic elastic plates through asymptotic homogenization. In particular, we consider soft inclusions and rigid inclusions with soft coatings embedded in a stiff matrix. The method provides a general expression for the dynamic surface density of the plate, which we compute analytically for circular inclusions or numerically for two-way ribbed plates. Through asymptotic homogenization, we find that band gaps related to in-plane propagating transversal waves occur for frequency intervals in which the effective surface density is negative. The same result is obtained via an asymptotic analysis of the Bloch-Floquet problem on a unit cell, showing the equivalence of the two approaches. Finally, we validate the method by comparing in several examples the predicted band gaps with those obtained from numerical Bloch-Floquet analyses on the real unit cell.
\end{abstract}

Keywords Metamaterials · Homogenization · Band gaps · Plates · Local resonance · Elasticity

Mathematics Subject Classification (2010) 74Q10 · 74H10

\section{Introduction}

Asymptotic homogenization is a mathematical tool widely employed in solid mechanics to describe the macroscopic behavior of periodically heterogeneous linearly elastic bodies $[5,6]$. The method, which in some cases provides analytical solutions, determines the homogenized properties of the periodic media based on the so-called cell problems.

C. Comi

claudia.comi@polimi.it

D. Faraci

david.faraci@polimi.it

J.-J. Marigo

jean-jacques.marigo@polytechnique.edu

1 Department of Civil and Environmental Engineering, Politecnico di Milano, Piazza Leonardo da Vinci 32, 20133 Milan, Italy

2 Laboratoire de Mécanique des Solides, École Polytechnique, Route de Saclay, 91128 Palaiseau, France 
This approach can also be used to study the static effective behavior of periodic plates of thickness $h$ and heterogeneities of size $a$. Starting from a three-dimensional formulation of the problem, one can study the asymptotic behavior of the plate as $h$ and $a$ tend to zero together, by fixing their ratio $[8,22,23]$. In addition to the previous case, Caillerie in [9] performed the asymptotic study for $h \rightarrow 0$ and $a \rightarrow 0$ separately, with either $a \rightarrow 0$ first or $h \rightarrow 0$ first, showing that the homogenized properties of the plate are different in the three cases. Here we will follow this latter option, assuming first a very thin plate and then taking the limit as the number of cells tends to infinity.

The homogenization problem can be simplified if the unit cell of the periodic plate is sufficiently thin with respect to the heterogeneities size, i.e., when it can be modelled as a Kirchhoff-Love plate. It is then possible, as done in [16] under static conditions, to perform the asymptotic analysis on the periodic plate starting directly from the equations of the structural theory. As discussed in [17] through several numerical analyses, this approach has limited applicability because in some cases the shear deformability of the plate cannot be disregarded even for slender unit cells. However, it will be sufficient for the present study which is focused on the low frequency dynamic behavior of metamaterial plates.

In the dynamic regime, periodic plates can act like locally resonant metamaterials (LRM), i.e., man-made materials which have the peculiar property of preventing the propagation of elastic waves in some frequency ranges (known as band gaps). This effect is due to the local resonance of some portions of the metamaterial which can trap the energy and attenuate wave propagation. Typically, LRM have a periodic or quasi-periodic structure consisting of a single material with local resonators inside the unit cells [14]; two-components materials, as studied, e.g., in [11, 19, 26]; or three components materials [10, 12, 21, 24].

The standard procedure to determine band gaps in LRM is to exploit their periodicity through the Bloch-Floquet theorem [7, 18] and to perform numerical dispersion analyses, see, e.g., [25]. Asymptotic homogenization is an alternative technique to analyze the dynamic behavior of solid LRM [3, 4, 13]. Under proper hypotheses on the geometry and on the materials constituting the unit cell, this method leads to analytical predictions of the band gaps, through the definition of a frequency-dependent effective mass density. This approach was developed in [11] for two-component LRM and in [12] for three-component ones.

In the present work, we extend the results obtained in [11] for bi-components solids to heterogeneous periodic thin plates.

For the plate homogenization, we first assume that the thickness $h$ is very small $(h \rightarrow$ 0 ), and, hence, that the Kirchhoff-Love theory applies. We then consider the size of the heterogeneity $a$ to be small with respect to the plate dimensions $(a \rightarrow 0)$.

When considering plates, several noteworthy differences with three dimensional solids arise.

Firstly, already in linear elastic isotropic homogeneous plates, the wave propagation is dispersive. In particular, using the Kirchhoff-Love theory one obtains the following dispersion relation between the wave frequency $\omega$ and the wave number $k$

$$
D k^{4}=\gamma \omega^{2},
$$

where the bending stiffness $D=E h^{3} / 12\left(1-v^{2}\right)$ depends on Young's modulus $E$ and Poisson's coefficient $v$, and the mass per unit area of the plate $\gamma=\rho h$ depends on the material mass density $\rho$. For very short-wavelength (high frequency) conditions, the dispersion relation (1) predicts an unbounded wave velocity that is physically unacceptable. Therefore the Kirchhoff-Love model itself can only be used in the low-frequency regime.

Secondly, the locally resonant effect, with corresponding band gap formation, can be achieved in periodic plates either by considering two or more different materials, namely 
soft inclusions inside a stiff matrix (Fig. 1a), or by considering a single material with a geometric variation (Fig. 2). In both cases, one can obtain a high contrast in the bending stiffness, and, in the present paper, we derive through homogenization the dispersion relation where the mass surface density $\gamma$ is replaced by an equivalent frequency-dependent mass surface density $\gamma^{0}(\omega)$. From this dispersion relation one can predict band gap formation in the range where the Kirchhoff-Love model is valid. The possibility to activate a locally resonant mechanism of band gap formation with a single material is a peculiar aspect of the meta-plates here studied.

Finally, we will show that in periodic plates, at difference from three-dimensional period solids, one can obtain band gaps at very low frequency by reducing the ratio $h / a$, independently of the lattice size.

The paper is organized as follows. After presenting the main hypotheses in Sect. 2, we study in Sect. 3 the transversal vibration of a periodic plate at a given frequency through asymptotic homogenization. In particular, we derive a general expression of the effective plate stiffness and dynamic surface density. In Sect. 4 the latter is analytically determined in closed form for circular inclusions and numerically computed for square ones.

In Sect. 5, we present the Bloch-Floquet problem related to the in-plane propagating transversal waves in the periodic plate. We prove that, in the limit of the homogenization analysis, the condition for the existence of band gaps given by the asymptotic Bloch-Floquet problem is exactly the same as the one obtained in Sect. 4, leading to a negative effective mass.

The homogenization approach is then extended in Sect. 6 to the case of ternary metamaterial plates.

Finally, in Sect. 7, we compare, via some examples, the band gaps predicted through homogenization by a negative effective mass, with those found numerically through BlochFloquet analysis, highlighting the validity and limitations of the proposed method.

Notation Vectors and second-order tensors are represented by bold face letters, while fourth-order tensors with blackboard bold letters. The symbol $\odot$ denotes the symmetric tensorial product. Summation over repeated indices is never implicitly assumed. Complex numbers and complex fields are denoted with sans-serif letters, e.g., i for $\sqrt{-1}$. The angular frequency $\omega$ is often referred to as frequency in the text for the sake of brevity.

\section{Vibration of Metamaterial Plates}

\subsection{Problem Setting}

We consider an elastic plate, with mid-surface $\Omega$ belonging to the $x_{1}-x_{2}$ plane, having nonconnected heterogeneities (also referred to as inclusions or fibers, $f$ ) periodically distributed in a connected matrix $(m)$. The inclusions will be assumed of cylindrical shape, independent from the out-of-plane coordinate $x_{3}$ as depicted in Fig. 1a and Fig. $1 \mathrm{~b}$.

We consider a large number of cells and we define a small parameter $\epsilon$ as the ratio between the typical dimensions $a$ of the cell and the size $\ell$ of the plate: $\epsilon=a / \ell$.

The in-plane periodicity is described by the Bravais lattice generated by the primitive vectors $\mathbf{a}_{1}$ and $\mathbf{a}_{2}$, which are represented in Fig. 1c on the unit cell $Y^{\epsilon}$ of the plate. In the same figure $Y_{m}^{\epsilon}$ and $Y_{f}^{\epsilon}$ denote the portions of the unit cell occupied by the matrix and the inclusion, respectively. 
Fig. 1 (a) Periodic plate composed by a matrix with cylindrical inclusions;

(b) mid-surface of the plate;

(c) unit cell; (d) reciprocal lattice and First Brillouin Zone

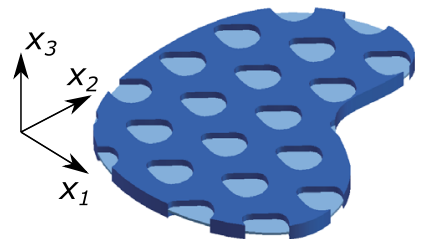

(a)

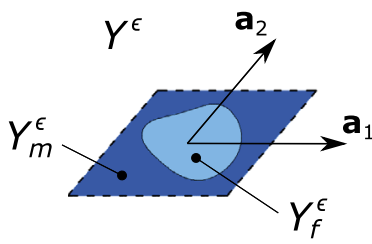

(c)

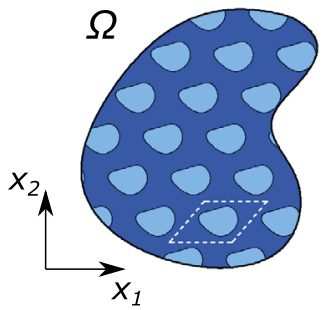

(b)

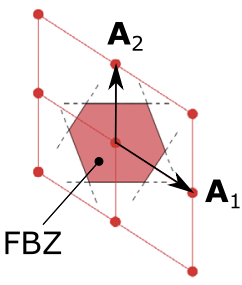

(d)
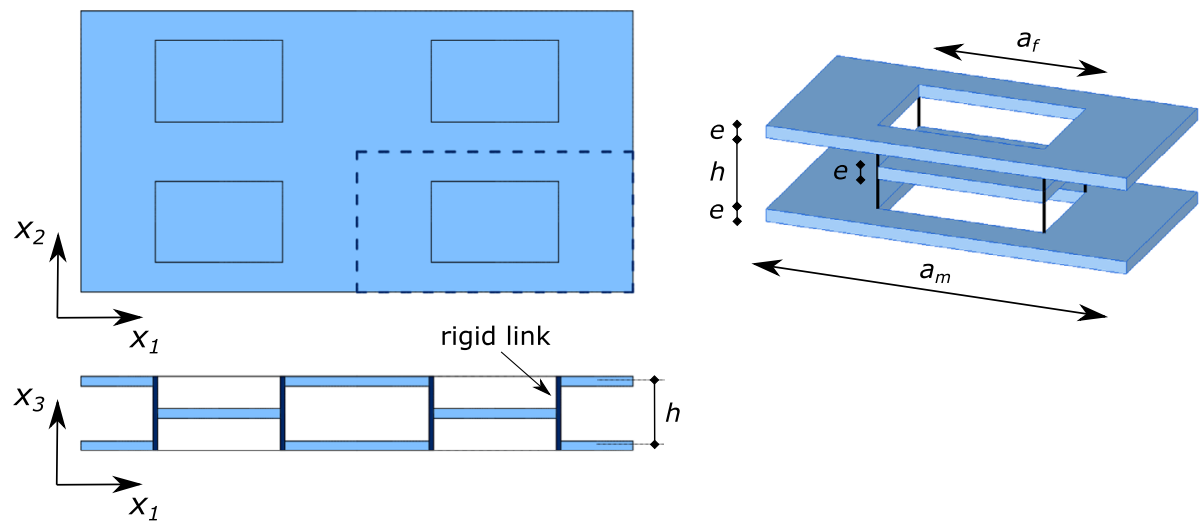

Fig. 2 Periodic plate made of a single material and different thicknesses: top view, vertical section and unit cell

Figure 1d shows the reciprocal lattice described by the vectors $\mathbf{A}_{1}$ and $\mathbf{A}_{2}$, defined as

$$
\mathbf{a}_{i} \cdot \mathbf{A}_{j}=2 \pi \delta_{i j}, \quad i, j \in\{1,2\}
$$

where $\delta_{i j}$ is the Kronecker symbol. In the same figure, the First Brillouin Zone (FBZ), i.e. the Wigner-Seitz unit cell of the reciprocal lattice [20], is shaded in red.

We assume that the thicknesses of the matrix, $h_{m}$, and of the inclusions, $h_{f}$, are much smaller than the unit cell size. In such a case, for low frequencies, the vibration of the plate can be studied using the Kirchhoff-Love model, neglecting the shear deformation and the rotational inertia of the plate.

Both constituent materials are modeled as linear-elastic and isotropic. 


\subsection{Governing Equations}

According to the Kirchhoff-Love formulation, the out of plane vibration of the considered heterogeneous plate at a given low frequency $\omega$ is governed by the differential equation

$$
\operatorname{div} \operatorname{div} \mathbf{M}^{\epsilon}+\gamma^{\epsilon} \omega^{2} w^{\epsilon}=0 \quad \text { in } \Omega,
$$

where $w^{\epsilon}$ is the out of plane displacement, div is the in-plane divergence operator, $\gamma^{\epsilon}$ is the surface density of the plate, i.e., the product of the mass density $\rho^{\epsilon}$ and the thickness $h^{\epsilon}$ (which vary periodically), and $\mathbf{M}^{\epsilon}$ is the moment tensor. For elastic materials this latter term can be expressed by the constitutive law

$$
\mathbf{M}^{\epsilon}=\mathbb{B}^{\epsilon}: \chi^{\epsilon}
$$

where $\chi^{\epsilon}$ is the curvature tensor of the plate defined as

$$
\chi^{\epsilon}=-\nabla \odot \nabla w^{\epsilon}
$$

For isotropic material the fourth-order stiffness tensor $\mathbb{B}^{\epsilon}$ in (4) has the following expression

$$
\mathbb{B}^{\epsilon}=D^{\epsilon}\left[\left(1-v^{\epsilon}\right) \mathbb{I}+v^{\epsilon} \mathbf{I} \otimes \mathbf{I}\right] .
$$

In (6), $D^{\epsilon}$ is the plate bending stiffness

$$
D^{\epsilon}=\frac{E^{\epsilon} h^{\epsilon 3}}{12\left(1-v^{\epsilon 2}\right)},
$$

with $E^{\epsilon}, v^{\epsilon}, h^{\epsilon}$ denoting the, periodically varying, Young's modulus, Poisson's ratio and plate thickness.

In this work the inclusion is supposed to be much softer than the matrix and to have a surface density of the same order of the matrix, namely the material properties in equations (3) and (4) are assumed as

$$
D^{\epsilon}=\left\{\begin{array}{ll}
D_{m} & \text { in } Y_{m}^{\epsilon} \\
\epsilon^{4} D_{f} & \text { in } Y_{f}^{\epsilon}
\end{array}, \quad \gamma^{\epsilon}=\left\{\begin{array}{ll}
\gamma_{m} & \text { in } Y_{m}^{\epsilon} \\
\gamma_{f} & \text { in } Y_{f}^{\epsilon}
\end{array},\right.\right.
$$

with $D_{m}$ and $\gamma_{m}$ of the same order of $D_{f}$ and $\gamma_{f}$, respectively. The particular scaling assumed between the stiffness will allow to obtain significant results through homogenization.

It is worth noting that, considering the plate made of a single material with the geometry of Fig. 2, with $a_{m} \sim a_{f}$, one has $D_{m} \sim E e h^{2} / 2\left(1-v^{2}\right)$ and $D_{f}=E e^{3} / 12\left(1-v^{2}\right)$. Hence, condition (8) is fulfilled for $e / h \sim \epsilon^{2}$.

\section{Homogenization Approach}

\subsection{Asymptotic Expansion}

In order to obtain the homogenized equation of motion we study the asymptotic behavior of the plate as $\epsilon \rightarrow 0$. 
Let $\mathbf{x}=x_{1} \mathbf{e}_{1}+x_{2} \mathbf{e}_{2}$ be the macroscopic coordinate, being $\mathbf{e}_{i}$ the unit vector in the $i$-th direction, and $\mathbf{y}=\mathbf{x} / \epsilon$ be the fast variable which lives in the re-scaled unit cell $Y=Y^{\epsilon} / \epsilon$. The two-scale asymptotic expansion of the out-of-plane displacement field reads

$$
w^{\epsilon}(\mathbf{x})=w^{0}\left(\mathbf{x}, \frac{\mathbf{x}}{\epsilon}\right)+\epsilon w^{1}\left(\mathbf{x}, \frac{\mathbf{x}}{\epsilon}\right)+\epsilon^{2} w^{2}\left(\mathbf{x}, \frac{\mathbf{x}}{\epsilon}\right)+\cdots,
$$

where the functions $w^{i}(\mathbf{x}, \mathbf{y})$ are defined on $\Omega \times \mathbb{R}^{2}$ and are $Y$-periodic with respect the fast variable $\mathbf{y}$. From (9) it is possible to derive by the chain rule

$$
\begin{aligned}
\nabla \odot \nabla w^{\epsilon}= & \epsilon^{-2} \nabla_{\mathbf{y}} \odot \nabla_{\mathbf{y}} w^{0}+\epsilon^{-1}\left(2 \nabla_{\mathbf{x}} \odot \nabla_{\mathbf{y}} w^{0}+\nabla_{\mathbf{y}} \odot \nabla_{\mathbf{y}} w^{1}\right) \\
& +\epsilon^{0}\left(\nabla_{\mathbf{x}} \odot \nabla_{\mathbf{x}} w^{0}+2 \nabla_{\mathbf{x}} \odot \nabla_{\mathbf{y}} w^{1}+\nabla_{\mathbf{y}} \odot \nabla_{\mathbf{y}} w^{2}\right)+\cdots,
\end{aligned}
$$

where $\nabla_{\mathbf{x}}$ and $\nabla_{\mathbf{y}}$ are the gradient with respect the variables $\mathbf{x}$ and $\mathbf{y}$, respectively.

By virtue of relationships (4),(5) and (10), the asymptotic expansion of the moment tensor starts at order -2 , namely:

$$
\mathbf{M}^{\epsilon}(\mathbf{x})=\epsilon^{-2} \mathbf{M}^{-2}\left(\mathbf{x}, \frac{\mathbf{x}}{\epsilon}\right)+\epsilon^{-1} \mathbf{M}^{-1}\left(\mathbf{x}, \frac{\mathbf{x}}{\epsilon}\right)+\mathbf{M}^{0}\left(\mathbf{x}, \frac{\mathbf{x}}{\epsilon}\right)+\cdots,
$$

where the tensor fields $\mathbf{M}^{i}(\mathbf{x}, \mathbf{y})$ are defined on $\Omega \times \mathbb{R}^{2}$ and are $Y$-periodic with respect $\mathbf{y}$. Thus it is possible to compute

$$
\begin{aligned}
\operatorname{div} \operatorname{div} \mathbf{M}^{\epsilon}= & \epsilon^{-4} \operatorname{div}_{\mathbf{y}} \operatorname{div}_{\mathbf{y}} \mathbf{M}^{-2} \\
& +\epsilon^{-3}\left(2 \operatorname{div}_{\mathbf{x}} \operatorname{div}_{\mathbf{y}} \mathbf{M}^{-2}+\operatorname{div}_{\mathbf{y}} \operatorname{div}_{\mathbf{y}} \mathbf{M}^{-1}\right) \\
& +\epsilon^{-2}\left(\operatorname{div}_{\mathbf{x}} \operatorname{div}_{\mathbf{x}} \mathbf{M}^{-2}+2 \operatorname{div}_{\mathbf{x}} \operatorname{div}_{\mathbf{y}} \mathbf{M}^{-1}+\operatorname{div}_{\mathbf{y}} \operatorname{div}_{\mathbf{y}} \mathbf{M}^{0}\right) \\
& +\epsilon^{-1}\left(\operatorname{div}_{\mathbf{x}} \operatorname{div}_{\mathbf{x}} \mathbf{M}^{-1}+2 \operatorname{div}_{\mathbf{x}} \operatorname{div}_{\mathbf{y}} \mathbf{M}^{0}+\operatorname{div}_{\mathbf{y}} \operatorname{div}_{\mathbf{y}} \mathbf{M}^{1}\right) \\
& +\epsilon^{0}\left(\operatorname{div}_{\mathbf{x}} \operatorname{div}_{\mathbf{x}} \mathbf{M}^{0}+2 \operatorname{div}_{\mathbf{x}} \operatorname{div}_{\mathbf{y}} \mathbf{M}^{1}+\operatorname{div}_{\mathbf{y}} \operatorname{div}_{\mathbf{y}} \mathbf{M}^{2}\right)+\cdots,
\end{aligned}
$$

where $\operatorname{div}_{\mathbf{x}}$ and $\operatorname{div}_{\mathbf{y}}$ are the divergence with respect the variables $\mathbf{x}$ and $\mathbf{y}$.

Substituting the expansions (9)-(12) in the governing equations (3)-(5) one obtains a sequence of differential problems associated to each order of the parameter $\epsilon$. Starting from order -4 , equation (3) reads

$$
\operatorname{div}_{\mathbf{y}} \operatorname{div}_{\mathbf{y}} \mathbf{M}^{-2}=0 \quad \text { in } \Omega \times Y,
$$

where

$$
\mathbf{M}^{-2}=\left\{\begin{array}{ll}
-\mathbb{B}_{m}: \nabla_{\mathbf{y}} \odot \nabla_{\mathbf{y}} w^{0} & \text { in } \Omega \times Y_{m} \\
\mathbf{0} & \text { in } \Omega \times Y_{f}
\end{array} .\right.
$$

Equations (13)-(14) together with the $Y$-periodicity imply that the $w^{0}$ must be independent on $\mathbf{y}$ in the matrix, namely

$$
w^{0}(\mathbf{x}, \mathbf{y})=W^{0}(\mathbf{x}) \quad \text { in } \Omega \times Y_{m} .
$$

The expansion of (3) at order -3 gives

$$
\operatorname{div}_{\mathbf{y}} \operatorname{div}_{\mathbf{y}} \mathbf{M}^{-1}=0 \quad \text { in } \Omega \times Y,
$$


where

$$
\mathbf{M}^{-1}= \begin{cases}-\mathbb{B}_{m}: \nabla_{\mathbf{y}} \odot \nabla_{\mathbf{y}} w^{1} & \text { in } \Omega \times Y_{m} \\ \mathbf{0} & \text { in } \Omega \times Y_{f}\end{cases}
$$

Similarly to the previous order, since $w^{1}$ must be periodic, we obtain that

$$
w^{1}(\mathbf{x}, \mathbf{y})=W^{1}(\mathbf{x}) \quad \text { in } \Omega \times Y_{m} .
$$

\subsection{The Matrix Cell Problem}

Focusing now on the order -2 of equation (3) we have to solve the differential equation

$$
\operatorname{div}_{\mathbf{y}} \operatorname{div}_{\mathbf{y}} \mathbf{M}^{0}=0 \quad \text { in } \Omega \times Y,
$$

where

$$
\mathbf{M}^{0}=\left\{\begin{array}{ll}
-\mathbb{B}_{m}:\left(\nabla_{\mathbf{x}} \odot \nabla_{\mathbf{x}} W^{0}+\nabla_{\mathbf{y}} \odot \nabla_{\mathbf{y}} w^{2}\right) & \text { in } \Omega \times Y_{m} \\
\mathbf{0} & \text { in } \Omega \times Y_{f}
\end{array} .\right.
$$

Since $W^{0}$ does not depend on $\mathbf{y}$, each component of $\nabla_{\mathbf{x}} \odot \nabla_{\mathbf{x}} W^{0}$ can be considered as a given eigen-curvature applied separately from the others. Due to linearity one has

$$
w^{2}(\mathbf{x}, \mathbf{y})=W^{2}(\mathbf{x})-\sum_{i, j=1}^{2} \frac{\partial^{2} W^{0}}{\partial x_{i} \partial x_{j}}(\mathbf{x}) \beta^{i j}(\mathbf{y}) \quad \text { in } \Omega \times Y_{m},
$$

where $\beta^{i j}(\mathbf{y})$ is the solution of the matrix cell problem

$$
\begin{cases}\Delta_{\mathbf{y}}^{2} \beta^{i j}=0 & \text { in } Y_{m} \\ \mathbf{M}^{i j}=\mathbb{B}_{m}:\left(-\nabla_{\mathbf{y}} \odot \nabla_{\mathbf{y}} \beta^{i j}+\mathbf{e}_{i} \odot \mathbf{e}_{j}\right) & \text { in } Y_{m} \\ M_{n}^{i j}=0 & \text { on } \partial Y_{m} \backslash \partial Y \\ Q^{i j}=0 & \text { on } \partial Y_{m} \backslash \partial Y \\ \beta^{i j}, M_{n}^{i j} \text { periodic } & \text { on } \partial Y \\ \nabla_{\mathbf{y}} \beta^{i j} \cdot \mathbf{n}, Q^{i j} \text { anti-periodic } & \text { on } \partial Y\end{cases}
$$

In (22) $\Delta_{\mathbf{y}}^{2}$ is the biharmonic operator with respect $\mathbf{y}, M_{n}^{i j}=\mathbf{n} \cdot \mathbf{M}^{i j} \cdot \mathbf{n}$ is the normal bending moment, $Q^{i j}=\left(\operatorname{div}_{\mathbf{y}} \mathbf{M}^{i j}\right) \cdot \mathbf{n}+\nabla_{\mathbf{y}}\left(\mathbf{t} \cdot \mathbf{M}^{i j} \cdot \mathbf{n}\right) \cdot \mathbf{t}$ is the Kirchhoff shear, $\mathbf{n}$ and $\mathbf{t}$ being the normal and the tangential unit vector at the boundaries $\partial Y_{f}$ and $\partial Y$. The solution of problem (22) is defined up to a constant, which represents a vertical rigid translation of the plate and can be fixed by enforcing

$$
\int_{Y_{m}} \beta^{i j}(\mathbf{y}) d \mathbf{y}=0 .
$$

Denoting by $\langle\bullet\rangle$ the average over $Y$ of the quantity $\bullet$, substituting (21) in (20) and taking the average, one obtains

$$
\left\langle\mathbf{M}^{0}\right\rangle(\mathbf{x})=\frac{1}{|Y|} \int_{Y} \mathbf{M}^{0}(\mathbf{x}, \mathbf{y}) d \mathbf{y}=-\mathbb{B}^{0}: \nabla_{\mathbf{x}} \odot \nabla_{\mathbf{x}} W^{0}(\mathbf{x}),
$$


where $\mathbb{B}^{0}$ is a fourth-order tensor of components

$B_{i j h k}^{0}=\frac{1}{|Y|} \int_{Y_{m}}\left(\mathbf{e}_{i} \odot \mathbf{e}_{j}-\nabla_{\mathbf{y}} \odot \nabla_{\mathbf{y}} \beta^{i j}\right): \mathbb{B}_{m}:\left(\mathbf{e}_{h} \odot \mathbf{e}_{k}-\nabla_{\mathbf{y}} \odot \nabla_{\mathbf{y}} \beta^{h k}\right) d \mathbf{y}, \quad i, j, h, k \in\{1,2\}$,

which represents the homogenized bending stiffness of the plate with cavities instead of the inclusions. From (25) it can be seen that $\mathbb{B}^{0}$ has all the minors and majors symmetries. Moreover, it is possible to prove that the effective bending stiffness is a positive definite tensor.

\subsection{The Inclusion Cell Problem}

At order 0 , equation (3) reads

$$
\operatorname{div}_{\mathbf{x}} \operatorname{div}_{\mathbf{x}} \mathbf{M}^{0}+2 \operatorname{div}_{\mathbf{x}} \operatorname{div}_{\mathbf{y}} \mathbf{M}^{1}+\operatorname{div}_{\mathbf{y}} \operatorname{div}_{\mathbf{y}} \mathbf{M}^{2}+\gamma \omega^{2} w^{0}=0 \quad \text { in } \Omega \times Y .
$$

Since the only non-vanishing moment in the inclusion is

$$
\mathbf{M}^{2}=-\mathbb{B}_{f}: \nabla_{\mathbf{y}} \odot \nabla_{\mathbf{y}} w^{0} \quad \text { in } \Omega \times Y_{f},
$$

the restriction of (26) on $\Omega \times Y_{f}$ provides the equation of motion in the fibers

$$
-D_{f} \Delta_{\mathbf{y}}^{2} w^{0}+\gamma_{f} \omega^{2} w^{0}=0 \quad \text { in } \Omega \times Y_{f}
$$

with prescribed boundary conditions which guarantee the continuity of displacements and rotations at the interface with the matrix, namely

$$
w^{0}=W^{0} \quad \text { and } \quad \nabla_{\mathbf{y}} w^{0} \cdot \mathbf{n}=0 \quad \text { on } \Omega \times \partial Y_{f} .
$$

Due to linearity the solution of (28)-(29) is given by

$$
w^{0}(\mathbf{x}, \mathbf{y})=W^{0}(\mathbf{x}) \eta(\mathbf{y}) \quad \text { in } \Omega \times Y_{f} .
$$

The function $\eta(\mathbf{y})$ is the solution of the inclusion cell problem

$$
\begin{cases}\Delta_{\mathbf{y}}^{2} \eta=\lambda^{4} \eta & \text { in } Y_{f} \\ \eta=1 & \text { on } \partial Y_{f} \\ \nabla_{\mathbf{y}} \eta \cdot \mathbf{n}=0 & \text { on } \partial Y_{f}\end{cases}
$$

with

$$
\lambda^{4}=\frac{\gamma_{f} \omega^{2}}{D_{f}}
$$

If $\omega$ is not an eigenfrequency of the inclusion fixed at its boundary, the solution of (31) is unique. When $\omega$ is an eigenfrequency there are two alternatives: either the eigenmodes are orthogonal to translations and problem (31) admits a (non unique) solution defined up to an arbitrary eigenmode, or some eigenmodes are non-orthogonal to translations and problem (31) has no solution. In this latter case from (28)-(30) one can deduce that it must be $W^{0}=0$. 


\subsection{The Effective Motion of the Plate}

Considering again the differential problem at order 0 given by (26), and taking the average over the unit cell $Y$ one obtains

$$
\operatorname{div}_{\mathbf{x}} \operatorname{div}_{\mathbf{x}}\left\langle\mathbf{M}^{0}\right\rangle+\left\langle\gamma w^{0}\right\rangle \omega^{2}=0 \quad \text { in } \Omega,
$$

since the average of all the other terms vanish after integrating by parts and considering the periodic boundary conditions on $\partial Y$.

From (15) and (30) one has

$$
\left\langle\gamma w^{0}\right\rangle=\gamma^{0} W^{0}
$$

where $\gamma^{0}$ is the effective surface density of the plate, defined as

$$
\gamma^{0}=\gamma_{m} \frac{\left|Y_{m}\right|}{|Y|}+\frac{\gamma_{f}}{|Y|} \int_{Y_{f}} \eta d \mathbf{y} .
$$

Introducing the static surface density $\gamma_{s t}$ and the mass contrast $\mu$ between the inclusion and the matrix, respectively defined as

$$
\gamma_{s t}=\gamma_{m} \frac{\left|Y_{m}\right|}{|Y|}+\gamma_{f} \frac{\left|Y_{f}\right|}{|Y|}, \quad \mu=\frac{\gamma_{f}\left|Y_{f}\right|}{\gamma_{m}\left|Y_{m}\right|}
$$

equation (35) can be effectively rewritten as

$$
\frac{\gamma^{0}}{\gamma_{s t}}=1+\frac{\mu}{1+\mu}\left(\frac{1}{\left|Y_{f}\right|} \int_{Y_{f}} \eta d \mathbf{y}-1\right) .
$$

The effective surface density defined in (35) does not depend on the particular shape of the unit cell, which can be arbitrary. As it can be seen from (37), $\gamma^{0}$ is determined by the static surface density $\gamma_{s t}$, the mass contrast $\mu$, and the particular inclusion considered. Substituting (24) and (34) into (33) one obtains the homogenized equation of motion of the plate

$$
-\operatorname{div}_{\mathbf{x}} \operatorname{div}_{\mathbf{x}}\left(\mathbb{B}^{0}: \nabla_{\mathbf{x}} \odot \nabla_{\mathbf{x}} W^{0}\right)+\gamma^{0} \omega^{2} W^{0}=0 \quad \text { in } \Omega .
$$

Note that the homogenized stiffness $\mathbb{B}^{0}$ is a constant tensor, while the effective surface density $\gamma^{0}$ depends on the frequency $\omega$, which intervenes, through $\lambda$, in the inclusion cell problem (31).

\subsection{The Band Gaps}

To analyze the propagation of transversal waves in the plane $x_{1}-x_{2}$, we consider a monochromatic wave of the form

$$
\mathrm{W}^{0}(\mathbf{x}, t)=W e^{\mathrm{i}(\mathbf{k} \cdot \mathbf{x}-\omega t)},
$$

where $W$ is the amplitude and $\mathbf{k}=k_{1} \mathbf{e}_{1}+k_{2} \mathbf{e}_{2}$ is the wavevector. Substituting (39) in the homogenized equation of vibration (38), non-trivial solutions can be found if and only if

$$
\mathbf{k} \odot \mathbf{k}: \mathbb{B}^{0}: \mathbf{k} \odot \mathbf{k}=\gamma^{0} \omega^{2} .
$$




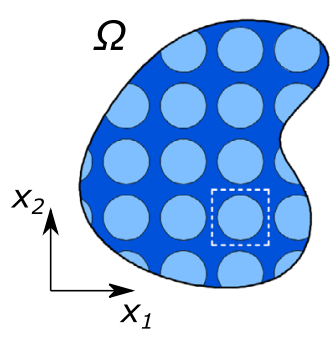

(a)

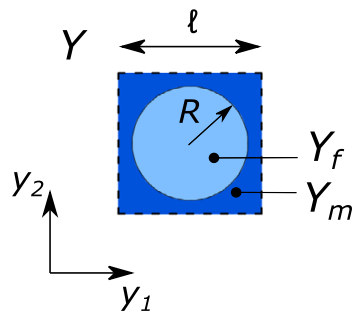

(b)

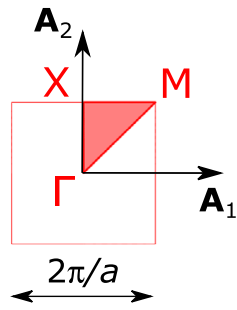

(c)

Fig. 3 (a) Plate with circular inclusions and square Bravais lattice; (b) re-scaled unit cell; (c) FBZ and IBZ (shaded in red)

Equation (40) defines implicitly the dispersion relation $\omega=\omega(\mathbf{k})$. Since $\mathbb{B}^{0}$ is positive defined, no real wavevector $\mathbf{k}$ can satisfy (40) when the RHS is negative. Therefore, the band gaps of the homogenized plate, i.e., the ranges of frequencies where transversal waves cannot propagate without being damped out, occur when the effective surface density $\gamma^{0}(\omega)$ is negative.

\section{Predictions of the Band Gaps}

\subsection{Plate with Circular Inclusions}

A close form expression of the effective surface density (37) can be obtained in the case of circular inclusions, as for the plate depicted in Fig. 3a. The re-scaled unit cell, as shown in Fig. $3 b$, is a square of side $\ell$, while the inclusion is given by

$$
Y_{f}=\left\{\mathbf{y} \in \mathbb{R}^{2} \text { s.t. }\|\mathbf{y}\| \leq R\right\} .
$$

Using polar coordinates $(r, \vartheta)$, solutions of problem (31) independent from $\vartheta$ can be searched in the form

$$
\eta(r)=a_{1} J_{0}(\lambda r)+a_{2} Y_{0}(\lambda r)+a_{3} I_{0}(\lambda r)+a_{4} K_{0}(\lambda r)
$$

where $J_{p}$ and $Y_{p}$ are the order $p$ Bessel functions of the first and second kind, $I_{p}$ and $K_{p}$ are the order $p$ modified Bessel functions of first and second kind, while $a_{i}(i=1,2,3,4)$ are constants to be determined.

The displacement $\eta$ and its directional derivatives must be bounded in a neighborhood of the origin, therefore it must be $a_{2}=a_{4}=0$. Enforcing the boundary conditions at $r=R$ we obtain the linear system

$$
\left\{\begin{array}{l}
a_{1} J_{0}(\lambda R)+a_{3} I_{0}(\lambda R)=1 \\
-a_{1} J_{1}(\lambda R)+a_{3} I_{1}(\lambda R)=0
\end{array},\right.
$$

which, in the case:

$$
d(\zeta)=J_{0}(\zeta) I_{1}(\zeta)+J_{1}(\zeta) I_{0}(\zeta) \neq 0
$$


admits the unique solution

$$
a_{1}=\frac{I_{1}(\lambda R)}{d(\lambda R)}, \quad a_{3}=\frac{J_{1}(\lambda R)}{d(\lambda R)}
$$

The function $d(\zeta)$ admits a countable number of roots

$$
\zeta_{1}=3.196, \quad \zeta_{2}=6.306, \quad \zeta_{3}=9.440, \quad \zeta_{4}=12.577, \quad \ldots,
$$

which define through (32) the frequencies

$$
\omega_{n}=\zeta_{n}^{2} \sqrt{\frac{D_{f}}{\gamma_{f} R^{4}}}, \quad n \in \mathbb{N}^{+} .
$$

The above frequencies $\omega_{n}$ are eigenfrequencies of the inclusion fixed at its boundary $(\eta=0$ and $\nabla_{\mathbf{y}} \eta \cdot \mathbf{n}=0$ on $\partial Y_{f}$ ) associated to radial eigenmodes. Therefore, if $\omega \neq \omega_{n}$ the radial solution exists and has the following expression

$$
\eta(r)=\frac{J_{0}(\lambda r) I_{1}(\lambda R)+J_{1}(\lambda R) I_{0}(\lambda r)}{J_{0}(\lambda R) I_{1}(\lambda R)+J_{1}(\lambda R) I_{0}(\lambda R)}
$$

If $\omega$ is an eigenfrequency $\omega=\omega_{n}$ such that $d\left(\zeta_{n}\right)=0$ (hence associated to a radial eigenmode of the inclusion fixed at its boundary) problem (31) has no solution.

When $\omega$ is an eigenfrequency of the inclusion fixed at its boundary such that $d\left(\zeta_{n}\right) \neq 0$ (i.e. corresponding to eigenmodes number different from zero $(p \neq 0))$, solutions of (31) not purely radial arise. However, as these eigenmodes $\eta_{p}$ are orthogonal to constants, i.e.,

$$
\int_{Y_{f}} \eta_{p} d \mathbf{y}=0
$$

they do not intervene in the definition of the effective surface density (see [11] for a general explanation for a three-dimensional solid).

Using (48) the mean value of $\eta(\mathbf{y})$ over the inclusion can be computed and reads

$$
\frac{1}{\left|Y_{f}\right|} \int_{Y_{f}} \eta d \mathbf{y}=\frac{4}{\lambda R} \frac{J_{1}(\lambda R) I_{1}(\lambda R)}{J_{0}(\lambda R) I_{1}(\lambda R)+J_{1}(\lambda R) I_{0}(\lambda R)} .
$$

By means of (37) one finally obtains the analytical expression of the effective surface density in the case of circular inclusions

$$
\frac{\gamma^{0}(\omega)}{\gamma_{s t}}=1+\frac{\mu}{1+\mu}\left(\frac{4}{\lambda R} \frac{J_{1}(\lambda R) I_{1}(\lambda R)}{J_{0}(\lambda R) I_{1}(\lambda R)+J_{1}(\lambda R) I_{0}(\lambda R)}-1\right) .
$$

From (32) and (47) (with $n=1$ ), one has $\lambda R=\zeta_{1} \sqrt{\omega / \omega_{1}}$. Using this relation in (51), the normalized effective mass $\gamma^{0} / \gamma_{s t}$ can be expressed as a function of $\omega / \omega_{1}$ and of the mass contrast $\mu$ only. Figure 4a shows the evolution of the normalized effective mass with $\omega / \omega_{1}$ for the case $\mu=5$. Dashed vertical lines are the asymptotes of the effective mass, while the orange shaded regions identify the frequency ranges in which $\gamma^{0}(\omega)<0$, i.e., the band gaps of the homogenized plate. Figure $4 \mathrm{~b}$ shows the width of the first three predicted band gaps as a function of the mass contrast. The opening frequencies are independent from $\mu$, 


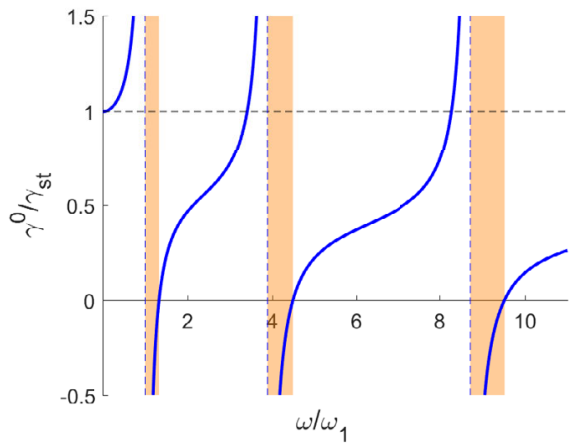

(a)

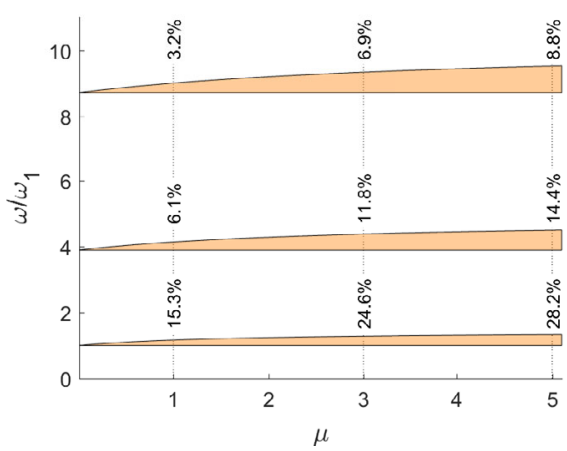

(b)

Fig. 4 Circular inclusion: (a) effective surface density $\gamma^{0} / \gamma_{s t}$ versus non-dimensional frequency $\omega / \omega_{1}$ for $\mu=5$; (b) non-dimensional band gaps frequency range versus mass contrast $\mu$

Fig. 5 (a) Two-way ribbed plate with square inclusions, (b) re-scaled unit cell

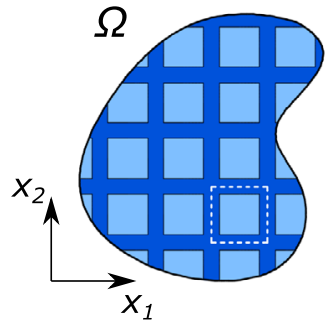

(a)

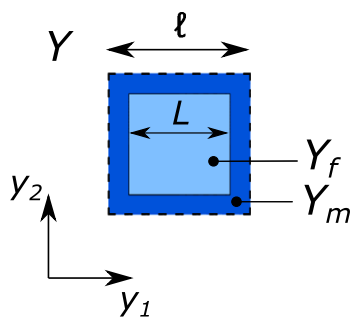

(b)

while the closure ones increase as the mass contrast becomes larger. The gap/mid-gap ratio, defined in terms of the opening and closure frequencies $\left(\omega_{o}\right.$ and $\left.\omega_{c}\right)$ of the band gap as

$$
B G[\%]=\frac{2\left(\omega_{c}-\omega_{o}\right)}{\omega_{c}+\omega_{o}} \cdot 100,
$$

is also reported in Fig. $4 \mathrm{~b}$ for $\mu=1,3,5$.

Remark As in the case of a two- or three-dimensional solid, the first band gap opens near the first eigenfrequency $\omega_{1}$ of the inclusion fixed at its boundary. However, there is a significant difference: while in the case of a non-structured solid the first eigenfrequency is proportional to the material celerity divided by the inclusion radius (see [11]), here the eigenfrequency depends on the same quantity (material celerity divided by the inclusion radius) multiplied by the ratio between thickness and radius of the inclusion $h / R$, Eq. (47). Therefore one can obtain a band gap at very low frequency also with small lattice dimensions by taking a small ratio $h / R$.

\subsection{Two-Way Ribbed Plate}

Another case of interest is the one, shown in Fig. 5a, of a two-way ribbed plate, which can represent, e.g., a waffle slab. The re-scaled unit cell, shown in Fig. 5b, has side $\ell$ with an inclusion $Y_{f}=[0, L] \times[0, L]$. 
Introducing the non-dimensional variable $\tilde{\mathbf{y}}=\mathbf{y} / L$ and the corresponding domain $\tilde{Y}_{f}=$ $Y_{f} / L$, the inclusion cell problem (31) is rewritten as

$$
\begin{cases}\Delta_{\tilde{\mathbf{y}}}^{2} \tilde{\eta}=\zeta^{4} \tilde{\eta} & \text { in } \tilde{Y}_{f} \\ \tilde{\eta}=1 & \text { on } \partial \tilde{Y}_{f} \\ \nabla_{\tilde{\mathbf{y}}} \tilde{\eta} \cdot \mathbf{n}=0 & \text { on } \partial \tilde{Y}_{f}\end{cases}
$$

where $\tilde{\eta}(\tilde{\mathbf{y}})=\eta(L \tilde{\mathbf{y}})$ and $\zeta=\lambda L$.

Since no simple analytical solutions are available for clamped square plates, the nondimensional problem (53) is numerically solved for increasing values of the parameter $\zeta$. The average value of $\tilde{\eta}$ numerically computed tends to infinity as $\zeta$ approaches one of the values

$$
\zeta_{1}=5.998, \quad \zeta_{2}=11.494, \quad \zeta_{3}=14.823, \quad \zeta_{4}=17.572, \quad \ldots,
$$

which define, similarly to the case of circular inclusions, the frequencies

$$
\omega_{n}=\zeta_{n}^{2} \sqrt{\frac{D_{f}}{\gamma_{f} L^{4}}}, \quad n \in \mathbb{N}^{+} .
$$

Equation (55) gives the eigenfrequencies of the inclusion, clamped at the boundary, associated to eigenmodes that are not orthogonal to constants.

For all the other values of $\zeta$, it is possible to evaluate numerically the effective surface density, which from (37) reads

$$
\frac{\gamma^{0}}{\gamma_{s t}}=1+\frac{\mu}{1+\mu}\left(\int_{\tilde{Y}_{f}} \tilde{\eta} d \tilde{\mathbf{y}}-1\right) .
$$

The effective surface density $\gamma^{0} / \gamma_{s t}$ is shown in Fig. 6a as a function of the non-dimensional frequency $\omega / \omega_{1}$ for mass contrast $\mu=5$. The shaded regions represent the band gaps. The variation of the first three band gaps with the mass contrast $\mu$ is shown in Fig. 6b; the values of the $B G$ ratios for $\mu=1,3,5$ are also reported. As for the circular inclusions, the opening frequencies are independent from $\mu$, while the closure ones increase monotonously with the mass contrast. The first two band gaps are comparable with those obtained in the case of circular inclusions, while the third one occurs at lower non-dimensional frequencies and has a smaller width (the $B G$ ratio is lower than $1 \%$ for $\mu \leq 5$ ).

\section{Bloch-Floquet Approach}

The common approach to derive the dispersion properties and the band gaps for transversal waves makes use of the Bloch-Floquet theorem [7, 18]. Following that approach, for any given real wavevector $\mathbf{k}=k_{1} \mathbf{e}_{1}+k_{2} \mathbf{e}_{2}$, one searches for the non-trivial solutions of the 


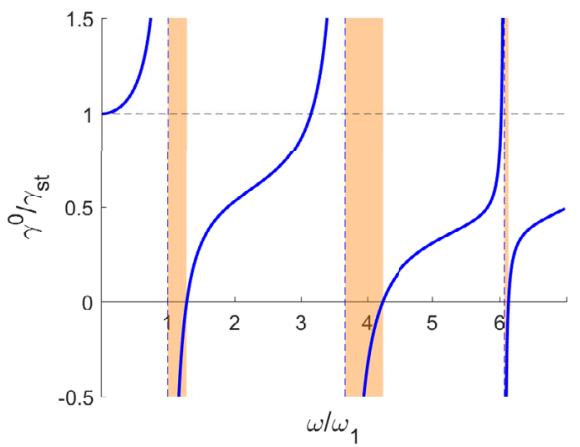

(a)

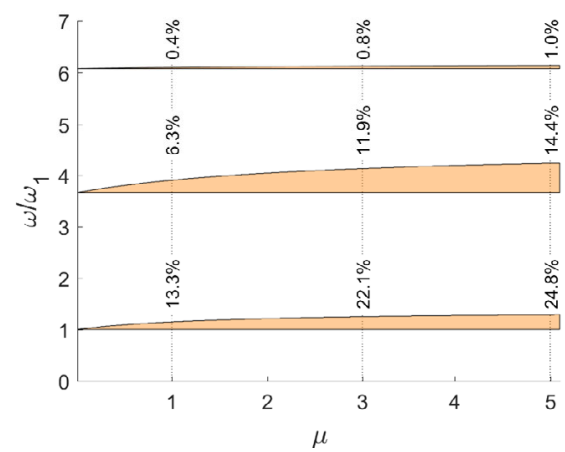

(b)

Fig. 6 Square inclusion: (a) effective surface density $\gamma^{0} / \gamma_{s t}$ versus non-dimensional frequency $\omega / \omega_{1}$ for $\mu=5$; (b) band gaps frequency range versus the mass contrast $\mu$

following problem

$$
\begin{cases}\operatorname{div} \operatorname{div} \mathrm{M}+\gamma^{\epsilon} \omega^{2} \mathrm{w}=0 & \text { in } Y^{\epsilon} \\ \mathrm{M}=-\mathbb{B}^{\epsilon}: \nabla \odot \nabla \mathrm{w} & \text { in } Y^{\epsilon} \\ \mathrm{w}\left(\mathbf{x}+\mathbf{a}_{i}\right)=\mathrm{w}(\mathbf{x}) e^{i \mathbf{k} \cdot \mathbf{a}_{i}} & \forall \mathbf{x} \text { on } \partial_{i}^{-} Y^{\epsilon}, i=1,2 \\ \nabla \mathrm{w}\left(\mathbf{x}+\mathbf{a}_{i}\right) \cdot \mathbf{n}=\nabla \mathrm{w}(\mathbf{x}) \cdot \mathbf{n} e^{i \mathbf{k} \cdot \mathbf{a}_{i}} & \forall \mathbf{x} \text { on } \partial_{i}^{-} Y^{\epsilon}, i=1,2 \\ \mathrm{M}_{n}\left(\mathbf{x}+\mathbf{a}_{i}\right)=\mathrm{M}_{n}(\mathbf{x}) e^{i \mathbf{k} \cdot \mathbf{a}_{i}} & \forall \mathbf{x} \text { on } \partial_{i}^{-} Y^{\epsilon}, i=1,2 \\ \mathrm{Q}\left(\mathbf{x}+\mathbf{a}_{i}\right)=\mathrm{Q}(\mathbf{x}) e^{i \mathbf{k} \cdot \mathbf{a}_{i}} & \forall \mathbf{x} \text { on } \partial_{i}^{-} Y^{\epsilon}, i=1,2\end{cases}
$$

where: $w$ is the complex out of plane displacement, $\mathrm{M}$ is the corresponding moment tensor, $\mathrm{M}_{n}=\mathbf{n} \cdot \mathrm{M} \cdot \mathbf{n}, \mathbf{Q}=\operatorname{div}(\mathrm{M}) \cdot \mathbf{n}+\nabla(\mathbf{t} \cdot \mathbf{M} \cdot \mathbf{n}) \cdot \mathbf{t}$ and $\partial_{i}^{-} Y^{\epsilon}$ is the side with outer normal $-\mathbf{n}$ of $Y^{\epsilon}$ in the $i$-th direction. Searching a periodic solution of the form

$$
\mathbf{w}(\mathbf{x})=\hat{\mathbf{w}}(\mathbf{x}) e^{i \mathbf{k} \cdot \mathbf{x}},
$$

problem (57) can be rewritten as

$$
\begin{cases}\operatorname{div} \operatorname{div} \hat{\mathrm{M}}+2 \mathrm{i} \operatorname{div} \hat{\mathrm{M}} \cdot \mathbf{k}-\hat{\mathrm{M}}: \mathbf{k} \odot \mathbf{k}+\gamma^{\epsilon} \omega^{2} \hat{\mathrm{w}}=0 & \text { in } Y^{\epsilon} \\ \hat{\mathrm{M}}=-\mathbb{B}^{\epsilon}:(\nabla \odot \nabla \hat{\mathrm{w}}+2 \mathrm{i} \nabla \hat{\mathrm{w}} \odot \mathbf{k}-\hat{\mathrm{w}} \mathbf{k} \odot \mathbf{k}) & \text { in } Y^{\epsilon} \\ \hat{\mathrm{w}} \text { and } \hat{\mathrm{M}}_{n} \text { periodic } & \text { on } \partial Y^{\epsilon} \\ (\nabla \hat{\mathrm{w}}+\mathrm{i} \hat{\mathrm{w}} \mathbf{k}) \cdot \mathbf{n} \text { and } \hat{\mathrm{Q}} \text { anti-periodic } & \text { on } \partial Y^{\epsilon}\end{cases}
$$

where $\hat{\mathrm{M}}_{n}=\mathbf{n} \cdot \hat{\mathrm{M}} \cdot \mathbf{n}$ and $\hat{\mathrm{Q}}=(\operatorname{div} \hat{\mathrm{M}}+\mathrm{i} \hat{\mathrm{M}} \cdot \mathbf{k}) \cdot \mathbf{n}+[\nabla(\mathbf{t} \cdot \hat{\mathrm{M}} \cdot \mathbf{n})+\mathrm{i}(\mathbf{t} \cdot \hat{\mathrm{M}} \cdot \mathbf{n}) \mathbf{k}] \cdot \mathbf{t}$.

By virtue of the spectral theorem [15], for given $\mathbf{k}$, the eigenfrequencies solutions of problem (59) constitute a sequence of real numbers which tends to infinity, the associated eigen-displacements constitute a subspace of finite dimensions and the eigen-spaces are orthogonal one to each other.

For each wave vector $\mathbf{k}$, the solution $\hat{w}$ and $\omega$ of problem (59) defines the amplitude and the frequency of propagating waves. If a solution exists for a given $\mathbf{k}$, it is also a solution for $\mathbf{k}+n \mathbf{A}_{1}+m \mathbf{A}_{2}$ (with $n, m \in \mathbb{N}^{+}$). This property follows from the definition (2) of the reciprocal lattice vectors $\mathbf{A}_{j}$. Therefore the analysis of the dispersion properties of the 
periodic plate can be restricted to $\mathbf{k}$ belonging to the First Brillouin Zone (FBZ) shown in Fig. 1.

Problem (59) can be numerically solved, e.g., by finite elements, by discretizing the unit cell and imposing the Bloch-Floquet boundary conditions. This leads to the dispersion plots $\omega(\mathbf{k})$; the intervals of frequency where no solutions exist define the bandgaps. This procedure will be used in Sect. 7 to obtain the results to be compared with the predictions given by the intervals of negative effective mass.

If the hypothesis listed in Sect. 2 are fulfilled, one can perform an asymptotic analysis to determine the homogenized Bloch-Floquet problem for the periodic plate. The homogenization, as developed in [11], allows to further highlight the relation between the Bloch-Floquet analysis and the study of the effective mass variation.

Considering again the fast variable $\mathbf{y}=\mathbf{x} / \epsilon$ and the re-scaled unit cell $Y=Y^{\epsilon} / \epsilon$, we define

$$
\hat{\mathrm{w}}^{\epsilon}(\mathbf{y})=\hat{\mathrm{w}}(\epsilon \mathbf{y}), \quad \hat{\mathrm{M}}^{\epsilon}(\mathbf{y})=\hat{\mathrm{M}}(\epsilon \mathbf{y}) \quad \text { in } Y .
$$

The problem (59) can hence be expressed as

$$
\begin{cases}\epsilon^{-2} \operatorname{div} \operatorname{div} \hat{\mathrm{M}}^{\epsilon}+2 \mathrm{i} \epsilon^{-1} \operatorname{div} \hat{\mathrm{M}}^{\epsilon}-\hat{\mathrm{M}}^{\epsilon}: \mathbf{k} \odot \mathbf{k}+\gamma^{\epsilon} \omega^{\epsilon 2} \hat{\mathrm{w}}^{\epsilon}=0 & \text { in } Y \\ \hat{\mathrm{M}}^{\epsilon}=-\epsilon^{-2} \mathbb{B}_{m}:\left(\nabla \odot \nabla \hat{\mathbf{w}}^{\epsilon}+2 \mathrm{i} \epsilon \nabla \hat{\mathrm{w}}^{\epsilon} \odot \mathbf{k}-\epsilon^{2} \hat{\mathbf{w}}^{\epsilon} \mathbf{k} \odot \mathbf{k}\right) & \text { in } Y_{m} \\ \hat{\mathrm{M}}^{\epsilon}=-\epsilon^{2} \mathbb{B}_{f}:\left(\nabla \odot \nabla \hat{\mathrm{w}}^{\epsilon}+2 \mathrm{i} \epsilon \nabla \hat{\mathrm{w}}^{\epsilon} \odot \mathbf{k}-\epsilon^{2} \hat{\mathrm{w}}^{\epsilon} \mathbf{k} \odot \mathbf{k}\right) & \text { in } Y_{f} \\ \hat{\mathrm{w}}^{\epsilon} \text { and } \hat{\mathrm{M}}_{n}^{\epsilon} \text { periodic } & \text { on } \partial Y \\ \left(\epsilon^{-1} \nabla \hat{\mathrm{w}}^{\epsilon}+\mathrm{i} \hat{\mathrm{w}}^{\epsilon} \mathbf{k}\right) \cdot \mathbf{n} \text { and } \hat{\mathbf{Q}}^{\epsilon} \text { anti-periodic } & \text { on } \partial Y\end{cases}
$$

where now $\nabla$ and div are the in-plane gradient and divergence with respect $\mathbf{y}, \hat{\mathrm{M}}_{n}^{\epsilon}=\mathbf{n} \cdot \hat{\mathrm{M}}^{\epsilon} \cdot \mathbf{n}$ and $\hat{\mathrm{Q}}^{\epsilon}=\left(\epsilon^{-1} \operatorname{div} \hat{\mathrm{M}}^{\epsilon}+\mathrm{i} \hat{\mathrm{M}}^{\epsilon} \cdot \mathbf{k}\right) \cdot \mathbf{n}+\left[\epsilon^{-1} \nabla\left(\mathbf{t} \cdot \hat{\mathrm{M}}^{\epsilon} \cdot \mathbf{n}\right)+\mathrm{i}\left(\mathbf{t} \cdot \hat{\mathrm{M}}^{\epsilon} \cdot \mathbf{n}\right) \mathbf{k}\right] \cdot \mathbf{t}$. We normalize the Bloch waves to have unit norm in $\mathrm{L}^{2}$ and we assume the following asymptotic expansions

$$
\left\{\begin{array}{l}
\hat{w}^{\epsilon}(\mathbf{y})=\hat{w}^{0}(\mathbf{y})+\epsilon \hat{\mathrm{w}}^{1}(\mathbf{y})+\epsilon^{2} \hat{\mathrm{w}}^{2}(\mathbf{y})+\cdots \\
\hat{\mathrm{M}}^{\epsilon}(\mathbf{y})=\hat{\mathrm{M}}^{0}(\mathbf{y})+\epsilon \hat{\mathrm{M}}^{1}(\mathbf{y})+\epsilon^{2} \hat{\mathrm{M}}^{2}(\mathbf{y})+\cdots \\
\omega^{\epsilon}=\omega+\cdots
\end{array},\right.
$$

where $\hat{w}^{0} \neq 0$ and the 0 -th order term of the frequency is called $\omega$ for simplicity sake. Note that the asymptotic expansion of the moment tensor should start at order -2 . However, it is possible to prove that $\hat{\mathrm{M}}^{-2}$ and $\hat{\mathrm{M}}^{-1}$ are null. Taking into account the constitutive law of the matrix, this implies

$$
\left\{\begin{array}{ll}
\nabla \odot \nabla \hat{\mathbf{w}}^{0}=\mathbf{0} & \text { in } Y_{m} \\
\nabla \odot \nabla \hat{\mathbf{w}}^{1}+2 \mathrm{i} \nabla \hat{\mathbf{w}}^{0} \odot \mathbf{k}=\mathbf{0} & \text { in } Y_{m}
\end{array},\right.
$$

which means that $\hat{w}^{0}$ and $\hat{w}^{1}$ are constant in the matrix, namely

$$
\hat{\mathbf{W}}^{0}(\mathbf{y})=\hat{\mathbf{W}}^{0} \quad \text { and } \quad \hat{\mathrm{W}}^{1}(\mathbf{y})=\hat{\mathrm{W}}^{1} \quad \text { in } Y_{m} .
$$

Substituting the expansions (62) into (61), one obtains a sequence of differential problems to be solved. At order -2 the problem reads

$$
\operatorname{div} \operatorname{div} \hat{\mathrm{M}}^{0}=0 \quad \text { in } Y,
$$


where

$$
\hat{\mathrm{M}}^{0}=\left\{\begin{array}{ll}
-\mathbb{B}_{m}:\left(\nabla \odot \nabla \hat{\mathrm{w}}^{2}-\hat{\mathrm{W}}^{0} \mathbf{k} \odot \mathbf{k}\right) & \text { in } Y_{m} \\
\mathbf{0} & \text { in } Y_{f}
\end{array} .\right.
$$

The linearity of (65) and (66) implies that

$$
\hat{\mathrm{w}}^{2}(\mathbf{y})=\hat{\mathrm{W}}^{2}+\hat{\mathrm{W}}^{0} \sum_{i, j=1}^{2} k_{i} k_{j} \beta^{i j}(\mathbf{y})
$$

being $\beta^{i j}(\mathbf{y})$ the solution of the matrix cell problem (22).

At order 0 the differential problem (61) reads

$$
\operatorname{div} \operatorname{div} \hat{\mathrm{M}}^{2}+2 \mathrm{i} \operatorname{div} \hat{\mathrm{M}}^{1} \cdot \mathbf{k}-\hat{\mathrm{M}}^{0} \mathbf{k} \odot \mathbf{k}+\gamma \omega^{2} \hat{\mathrm{w}}^{0}=0 \quad \text { in } Y .
$$

Since $\hat{\mathrm{M}}^{0}$ and $\hat{\mathrm{M}}^{1}$ are zero in the fibers, restricting (68) over $Y_{f}$ we obtain the differential problem

$$
\begin{cases}-D_{f} \Delta^{2} \hat{\mathbf{w}}^{0}+\gamma_{f} \omega^{2} \hat{\mathbf{w}}^{0}=0 & \text { in } Y_{f} \\ \hat{\mathbf{w}}^{0}=\hat{\mathbf{W}}^{0} & \text { on } \partial Y_{f} \\ \nabla \hat{\mathbf{w}}^{0} \cdot \mathbf{n}=0 & \text { on } \partial Y_{f}\end{cases}
$$

The solution of (69) is given by

$$
\hat{\mathbf{w}}^{0}(\mathbf{y})=\hat{\mathbf{W}}^{0} \eta(\mathbf{y}) \quad \text { in } Y_{f},
$$

where $\eta(\mathbf{y})$ is the solution of the inclusion cell problem (31).

Considering again equation (68), and taking its average over the unit cell $Y$, one obtains

$$
-\left\langle\hat{\mathrm{M}}^{0}\right\rangle: \mathbf{k} \odot \mathbf{k}+\left\langle\gamma \hat{\mathrm{w}}^{0}\right\rangle \omega^{2}=0 .
$$

By means of (64), (66), (67) and (70) it is possible to evaluate

$$
\left\langle\hat{\mathrm{M}}^{0}\right\rangle=\hat{\mathrm{W}}^{0} \mathbb{B}^{0}: \mathbf{k} \odot \mathbf{k}, \quad\left\langle\gamma \hat{\mathrm{W}}^{0}\right\rangle=\gamma^{0} \hat{\mathrm{W}}^{0},
$$

where $\mathbb{B}^{0}$ and $\gamma^{0}$ are, respectively, the homogenized stiffness tensor and the effective surface density of the plate given by (25) and (35). Substituting (72) into (71), we obtain the homogenized Bloch-Floquet equation

$$
\left(-\mathbf{k} \odot \mathbf{k}: \mathbb{B}^{0}: \mathbf{k} \odot \mathbf{k}+\gamma^{0} \omega^{2}\right) \hat{\mathrm{W}}^{0}=0,
$$

that is the dispersion relation for in-plane propagating transversal waves. Since $\hat{\mathbf{W}}^{0} \neq 0$, equation (73) coincides with (40), which was derived from the homogenization of transversal plate vibrations. This shows that, in the limit of the asymptotic analysis, the two approaches are equivalent and the prediction of the band gaps based on the Bloch-Floquet homogenized theory corresponds to intervals of negative effective surface density. It is worth to underline that this result holds true at the first order approximation when considering soft inclusions within a stiff matrix. The equivalence might not remain true when considering higher order approximations in the expansion, as shown for a different problem in [1]. 
Fig. 7 (a) Three-constituents plate with coated circular inclusions, (b) re-scaled unit cell

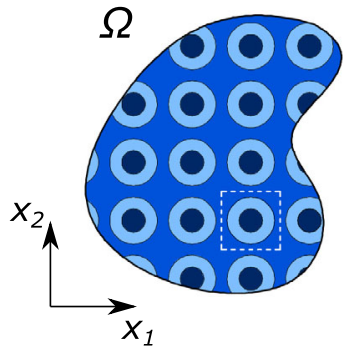

(a)

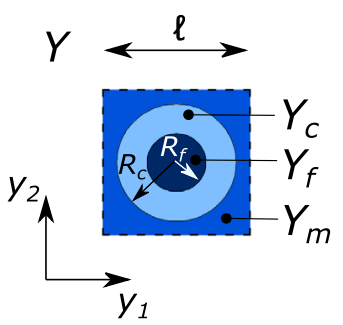

(b)

\section{Extension to Ternary Locally Resonant Plate}

The homogenization approach described in Sect. 3 can be easily extended to the case of a three constituents metamaterial plate. As shown in Fig. 7a, we consider a connected matrix $(m)$ with rigid circular inclusions $(f)$ coated by a soft material $(c)$. The material properties of the three constituent materials are

$$
D^{\epsilon}=\left\{\begin{array}{ll}
D_{m} & \text { in } Y_{m}^{\epsilon} \\
\epsilon^{4} D_{c} & \text { in } Y_{c}^{\epsilon}
\end{array}, \quad \gamma^{\epsilon}= \begin{cases}\gamma_{m} & \text { in } Y_{m}^{\epsilon} \\
\gamma_{c} & \text { in } Y_{c}^{\epsilon} \\
\gamma_{f} & \text { in } Y_{f}^{\epsilon}\end{cases}\right.
$$

being $D_{m}, D_{c}$ and $\gamma_{m}, \gamma_{c}, \gamma_{f}$ of the same order of magnitude. The re-scaled unit cell of the plate is a square of side $\ell$, shown in Fig. 7b, with a circular inclusion of radius $R_{f}$ and an annular coating of external radius $R_{c}$.

The asymptotic expansion of the out of plane displacement is the same assumed in (9). As occurs in Sect. 3.1, the 0-th order displacement in the matrix does not depend on the fast variable, namely

$$
w^{0}(\mathbf{x}, \mathbf{y})=W_{m}^{0}(\mathbf{x}) \quad \text { in } \Omega \times Y_{m} .
$$

The derivation of the effective bending stiffness and the matrix cell problem follow exactly the same path explained in Sect. 3.2 and leads to the same results.

Considering the equation of motion at order 0 in the coating, as done in Sect. 3.3, one obtains

$$
-D_{c} \Delta_{\mathbf{y}} w^{0}+\gamma_{c} \omega^{2} w^{0}=0 \quad \text { in } \Omega \times Y_{c},
$$

with proper boundary conditions in order to guarantee the continuity of displacement and rotations with the matrix and the inclusion, namely

$$
w^{0}=W_{m}^{0} \quad \text { on } \partial Y_{c} \backslash \partial Y_{f}, \quad w^{0}=W_{f}^{0} \quad \text { on } \partial Y_{f} \quad \text { and } \quad \nabla_{\mathbf{y}} w^{0} \cdot \mathbf{n}=0 \quad \text { on } \partial Y_{c},
$$

where $W_{f}^{0}(\mathbf{x})$ is the displacement of the rigid inclusion, independent from $\mathbf{y}$ for radial solution of equation (76). The solution of (76) is assumed to be in the form

$$
w^{0}(\mathbf{x}, \mathbf{y})=W_{m}^{0}(\mathbf{x}) \eta(\mathbf{y}) \quad \text { in } \Omega \times Y_{c},
$$


where $\eta(\mathbf{y})$ must satisfy the inclusion cell problem

$$
\begin{cases}\Delta_{\mathbf{y}}^{2} \eta=\lambda^{4} \eta & \text { in } Y_{c} \\ \eta=1 & \text { on } \partial Y_{c} \backslash \partial Y_{f} \\ \eta=W_{f}^{0} / W_{m}^{0} & \text { on } \partial Y_{f} \\ \nabla_{\mathbf{y}} \eta \cdot \mathbf{n}=0 & \text { on } \partial Y_{c}\end{cases}
$$

and

$$
\lambda^{4}=\frac{\gamma_{c} \omega^{2}}{D_{c}} .
$$

Radial solutions of problem (79) can be searched in the form

$$
\eta(r)=f(r)+\frac{W_{f}^{0}}{W_{m}^{0}} g(r),
$$

where $f$ and $g$ satisfy the differential problems

$$
\left\{\begin{array} { l l } 
{ \Delta _ { \mathbf { y } } ^ { 2 } f = \lambda ^ { 4 } f } & { \text { in } Y _ { c } } \\
{ f = 1 } & { \text { for } r = R _ { c } } \\
{ f = 0 } & { \text { for } r = R _ { f } } \\
{ \frac { \partial f } { \partial r } = 0 } & { \text { for } r = R _ { c } , R _ { f } }
\end{array} \quad \text { and } \quad \left\{\begin{array}{ll}
\Delta_{\mathbf{y}}^{2} g=\lambda^{4} g & \text { in } Y_{c} \\
g=0 & \text { for } r=R_{c} \\
g=1 & \text { for } r=R_{f} \\
\frac{\partial g}{\partial r}=0 & \text { for } r=R_{c}, R_{f}
\end{array} .\right.\right.
$$

Therefore, $f$ and $g$ have general expressions

$$
\begin{aligned}
& f(r)=a_{1} J_{0}(\lambda r)+a_{2} Y_{0}(\lambda r)+a_{3} I_{0}(\lambda r)+a_{4} K_{0}(\lambda r), \\
& g(r)=b_{1} J_{0}(\lambda r)+b_{2} Y_{0}(\lambda r)+b_{3} I_{0}(\lambda r)+b_{4} K_{0}(\lambda r),
\end{aligned}
$$

where $a_{i}, b_{i}(i=1,2,3,4)$ are constants to be determined from the boundary conditions. The radial solution exists iff the matrix

$$
\mathbf{C}=\left(\begin{array}{cccc}
J_{0}\left(\lambda R_{c}\right) & Y_{0}\left(\lambda R_{c}\right) & I_{0}\left(\lambda R_{c}\right) & K_{0}\left(\lambda R_{c}\right) \\
J_{0}\left(\lambda R_{f}\right) & Y_{0}\left(\lambda R_{f}\right) & I_{0}\left(\lambda R_{f}\right) & K_{0}\left(\lambda R_{f}\right) \\
J_{1}\left(\lambda R_{c}\right) & Y_{1}\left(\lambda R_{c}\right) & -I_{1}\left(\lambda R_{c}\right) & K_{1}\left(\lambda R_{c}\right) \\
J_{1}\left(\lambda R_{f}\right) & Y_{1}\left(\lambda R_{f}\right) & -I_{1}\left(\lambda R_{f}\right) & K_{1}\left(\lambda R_{f}\right)
\end{array}\right)
$$

is not singular, which corresponds to the case in which $\omega$ is not an eigenfrequency of the clamped-clamped annular coating. In such a case, the coefficients in (83) are the solutions of the linear systems

$$
\mathbf{C}\left(\begin{array}{l}
a_{1} \\
a_{2} \\
a_{3} \\
a_{4}
\end{array}\right)=\left(\begin{array}{l}
1 \\
0 \\
0 \\
0
\end{array}\right) \quad \text { and } \quad \mathbf{C}\left(\begin{array}{l}
b_{1} \\
b_{2} \\
b_{3} \\
b_{4}
\end{array}\right)=\left(\begin{array}{l}
0 \\
1 \\
0 \\
0
\end{array}\right) .
$$

The radial solution (81) is therefore determined up to the ratio $W_{f}^{0} / W_{m}^{0}$, which can be determined enforcing the global dynamic equilibrium equation on the rigid inclusion

$$
-D_{c} \int_{\partial Y_{f}} \nabla_{\mathbf{y}}\left(\Delta_{\mathbf{y}} w^{0}\right) \cdot \mathbf{n} d s+\gamma_{f}\left|Y_{f}\right| \omega^{2} W_{f}^{0}=0
$$


from which is possible to express

$$
\frac{W_{f}^{0}}{W_{m}^{0}}=\frac{a_{1} J_{1}\left(\lambda R_{f}\right)+a_{2} Y_{1}\left(\lambda R_{f}\right)}{\frac{\gamma_{f}}{\gamma_{c}} \frac{\lambda R_{f}}{4}-b_{1} J_{1}\left(\lambda R_{f}\right)-b_{2} Y_{1}\left(\lambda R_{f}\right)}
$$

As done in Sect. 3.4, one can obtain the effective equation of motion

$$
-\operatorname{div}_{\mathbf{x}} \operatorname{div}_{\mathbf{x}}\left(\mathbb{B}^{0}: \nabla_{\mathbf{x}} \odot \nabla_{\mathbf{x}} W_{m}^{0}\right)+\gamma^{0} \omega^{2} W_{m}^{0}=0 \quad \text { in } \Omega,
$$

where $\mathbb{B}^{0}$ is the effective bending stiffness tensor given by (25) and $\gamma^{0}(\omega)$ is the frequency dependent effective surface density having expression

$$
\frac{\gamma^{0}(\omega)}{\gamma_{s t}}=1+\frac{\mu_{c}}{1+\mu_{c}+\mu_{f}}\left(\frac{1}{\left|Y_{c}\right|} \int_{Y_{c}} \eta d \mathbf{y}-1\right)+\frac{\mu_{f}}{1+\mu_{c}+\mu_{f}}\left(\frac{W_{f}^{0}}{W_{m}^{0}}-1\right),
$$

where is introduced the static surface density

$$
\gamma_{s t}=\gamma_{m} \frac{\left|Y_{m}\right|}{|Y|}+\gamma_{c} \frac{\left|Y_{c}\right|}{|Y|}+\gamma_{f} \frac{\left|Y_{f}\right|}{|Y|}
$$

and the mass contrast between the inner phases and the matrix

$$
\mu_{c}=\frac{\gamma_{c}\left|Y_{c}\right|}{\gamma_{m}\left|Y_{m}\right|} \quad \text { and } \quad \mu_{f}=\frac{\gamma_{f}\left|Y_{f}\right|}{\gamma_{m}\left|Y_{m}\right|} .
$$

The average of the solution of (79) on the coating figuring in (89) reads

$$
\begin{aligned}
& \frac{1}{\left|Y_{c}\right|} \int_{Y_{c}} \eta d \mathbf{y}=\frac{4}{\lambda\left(R_{c}^{2}-R_{f}^{2}\right)} {\left[\left(a_{1}+b_{1} \frac{W_{f}^{0}}{W_{m}^{0}}\right)\left(R_{f} J_{1}\left(\lambda R_{f}\right)-R_{c} J_{1}\left(\lambda R_{c}\right)\right)+\right.} \\
&\left.+\left(a_{2}+b_{2} \frac{W_{f}^{0}}{W_{m}^{0}}\right)\left(R_{f} Y_{1}\left(\lambda R_{f}\right)-R_{c} Y_{1}\left(\lambda R_{c}\right)\right)\right] .
\end{aligned}
$$

\section{Examples}

To validate the homogenization technique presented in Sect. 3, Sect. 5 and Sect. 6, we compare the band gaps prediction with those obtained by numerical Bloch-Floquet analysis on the real unit cell of the periodic plate.

\subsection{Two-Constituents Metamaterial Plates}

\subsubsection{Materials Selection}

The proposed method relies mainly on two hypotheses: thin unit cell and high stiffness contrast between the two phases. In particular, introducing the ratio $\xi=D_{m} / \epsilon^{4} D_{f}$ between the matrix and inclusion plate bending stiffnesses, the predicted band gaps are more and more accurate as $\xi \rightarrow+\infty$. This suggests the employment of a very stiff material for the matrix (e.g., a metal) and a soft one for the inclusions (e.g., an elastomer). Considering that 


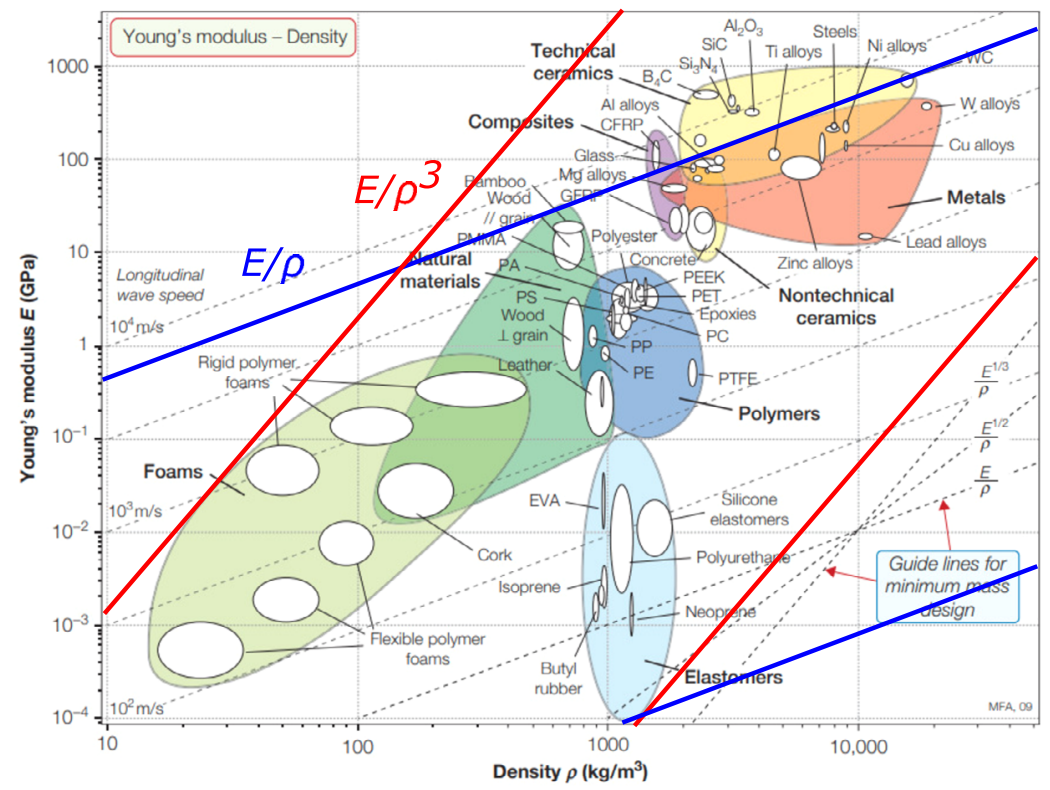

Fig. 8 Ashby's chart of Young's modulus $E$ against mass density $\rho$, from [2]

stiff materials are usually heavier than soft ones, this choice strongly affects the width of the band gaps. As discussed in Sect. 4, the gap-midgap ratio decreases as the mass contrast of the inclusion with respect to the matrix becomes smaller.

Following the approach for the material selection proposed by [2], one can define a material index which depends on the material properties only and which intervenes in the considered problem, characterized by the above competing requirements.

Assuming that both component materials have similar Poisson's ratio $\left(v_{m} \simeq v_{f}\right)$ we can consider the product:

$$
\xi \mu^{3} \simeq \frac{E_{m} / \rho_{m}^{3}}{E_{f} / \rho_{f}^{3}} \frac{\left|Y_{f}\right|^{3}}{\left|Y_{m}\right|^{3}},
$$

which does not depend on the thicknesses and should be maximized. One can hence consider the material index $E / \rho^{3}$, appearing in (93), which has to be maximized for the matrix and minimized for the inclusions.

To this purpose, we refer to the Ashby's chart, shown in Fig. 8, which reports Young's modulus $E$ versus the mass density $\rho$ for different materials. In this bi-logarithmic plot, the red straight lines identify the materials for which $E / \rho^{3}$ is maximized (rigid polymer foams) and minimized (elastomers). Note that a different choice of the material index, e.g., $E / \rho$ in blue, would lead to the same minimizing materials, but different maximizing ones (metals).

In the following examples, we consider inclusions made of ethylene-propylene-diene monomer (EPDM), while for the matrix we consider two solutions: aluminium and rigid polyurethane (PU) foam. Typical properties of the employed materials are listed in Table 1 . 
Table 1 Materials properties

\begin{tabular}{llll}
\hline & $\rho\left[\mathrm{kg} / \mathrm{m}^{3}\right]$ & $E[\mathrm{MPa}]$ & $v[-]$ \\
\hline Rigid PU foam & 800 & 1600 & 0.3 \\
Aluminium & 2500 & 70000 & 0.34 \\
EPDM & 1400 & 6 & 0.49 \\
Epoxy & 1180 & 3600 & 0.37 \\
Rubber & 1300 & 0.118 & 0.47 \\
Lead & 11340 & 14000 & 0.37 \\
\hline
\end{tabular}

\subsubsection{Plate with Circular Inclusions}

As the first example, consider a plate of uniform thickness $h$, with circular inclusions of radius $R=0.45 \ell$ characterized by a square lattice, like the one shown in Fig. 3.

The (rotated) plot of the effective surface density $\gamma^{0} / \gamma_{s t}$ against the non-dimensional frequency $\omega / \omega_{1}$, where $\omega_{1}$ is given by (47) for $n=1$, is reported on the left in Fig. 9a for an aluminium matrix, and in Fig. $9 \mathrm{~b}$ for a rigid PU foam matrix. In both cases, the inclusion is made of EPDM.

The same figures, on the right, show the dispersion plots obtained by a Bloch-Floquet analysis on the unit cell of the plate (with $\Gamma, X$ and $M$ being the vertex of the Irreducible Brillouin Zone (IBZ), as in Fig. 3c).

In the case of aluminium matrix, Fig. 9a, the stiffness contrast is very high $\left(\xi \simeq 10^{4}\right)$ and the first three band gaps (shaded in orange) are correctly predicted. The dispersion plot also shows the presence of a flat mode within the third band gap. As explained in detail in [11], this can be taken into account also in the homogenization procedure by considering the eigenfrequencies of the clamped inclusion corresponding to modes orthogonal to constants.

When the matrix is made of rigid PU foam, Fig. 9b, only the first interval of negative mass density actually corresponds to a band gap in the dispersion plot. This is due to the insufficient stiffness contrast $\left(\xi=2.23 \cdot 10^{2}\right)$ that affects the main hypothesis of the homogenization method. However, as expected due to the material selection, the $B G$ ratio of the first band gap is higher with respect to the previous case. That is because of the mass contrast, which is $\mu=0.98$ when considering an aluminum matrix and $\mu=3.06$ when considering a PU foam matrix.

\subsubsection{Two-Way Ribbed Plate}

The second example concerns a two-way ribbed plate, as depicted in Fig. 5, with a square inclusion of side $L=0.8 \ell$. The matrix is made of rigid PU foam, while the inclusions are made of EPDM. The IBZ of the periodic plate is again the one shown in Fig. 3c. Frequencies are normalized with respect the first resonant frequency of the inclusions provided by (55) for $n=1$.

As discussed in the previous example, if the plate has uniform thickness $h$, the stiffness contrast $\xi=2.23 \cdot 10^{2}$ is sufficient to predict only the first band gap, see Fig. 10a. However, one can increase the thickness of the matrix to increase its bending stiffness. Choosing $h_{m}=2.5 h_{f}=2.5 h$, that means $\xi=3.43 \cdot 10^{3}$, as shown in Fig. 10b, also the second interval of negative effective mass corresponds to the second band gap in the dispersion plot. It should be noticed that in this way the width of the band gaps decreases since the mass contrast reduces from $\mu=3.11$ (Fig. 10a) to $\mu=1.24$ (Fig. 10b). 

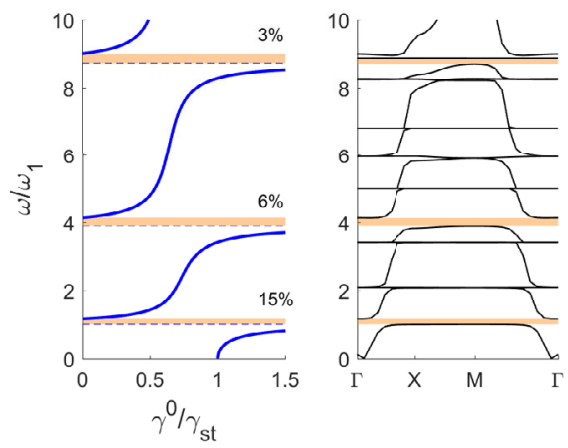

(a)
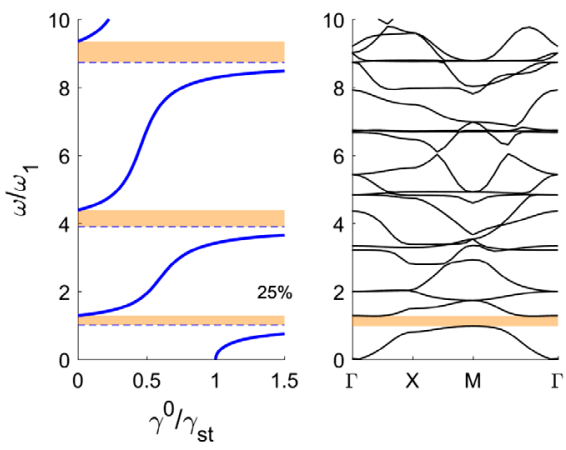

(b)

Fig. 9 Comparison between band gaps predicted through homogenization and the band gaps given by BlochFloquet analysis for circular inclusions of radius $R=0.45 \ell$ and uniform thickness $h$. Inclusions: EPDM; matrix: (a) aluminium, (b) rigid PU foam
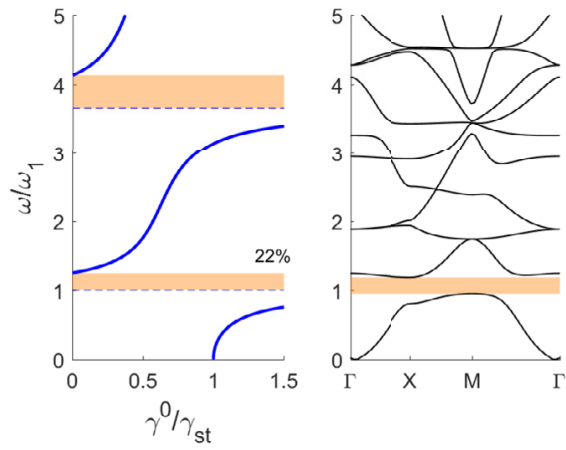

(a)
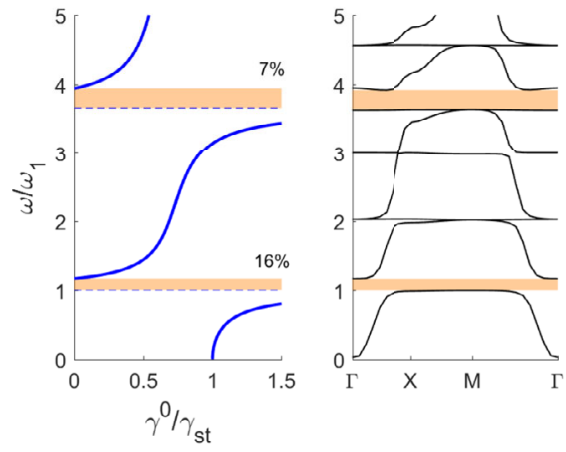

(b)

Fig. 10 Comparison between band gaps predicted through homogenization and band gaps given by BlochFloquet analysis for square inclusions of side $L=0.8 \ell$; matrix of rigid PU foam, inclusions of EPDM. (a) Uniform thickness $h$; (b) $h_{m}=2.5 h_{f}=2.5 h$

\subsection{Three-Constituents Metamaterial Plates}

\subsubsection{Materials Selection}

The presence of three different constituent materials allows to obtain larger band gaps. Actually, the requirement of the stiffness contrast, for the validity of the asymptotic analysis, and that on the mass contrast, for wide band gaps, concern different materials and are no more in conflict. A very compliant coating within a stiff matrix ensures the proper stiffness contrast while a heavy, stiff material for the inclusion enables to have a wide band gap.

In the example we will use the same materials considered in [12]: epoxy matrix, rubber coating and lead inclusions. Typical properties of these materials are reported in Table 1 . 


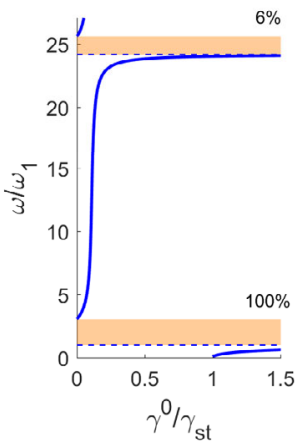

(a)

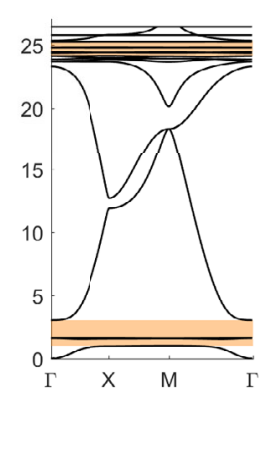

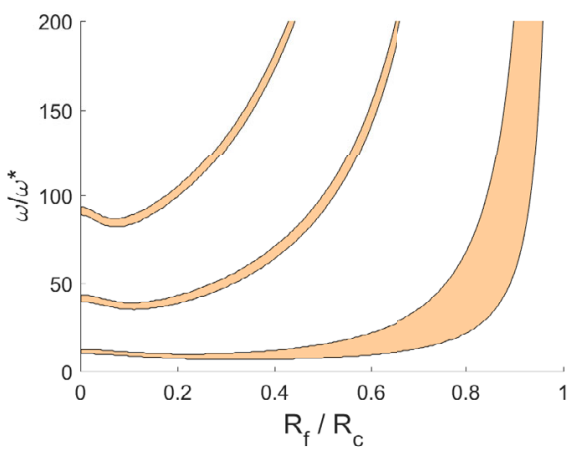

(b)

Fig. 11 Three phase plate: (a) comparison between band gaps predicted through homogenization and band gaps given by Bloch Floquet analysis $\left(R_{C}=0.45 \ell, R_{f} / R_{c}=0.77\right)$; (b) frequency ranges of the first three band gaps against the ratio $R_{f} / R_{c}$ for the case $R_{c}=0.45 \ell$

\subsubsection{Plate with Circular Inclusions and Annular Coatings}

We refer to the ternary plate shown in Fig. 7 of uniform thickness $h$, made of an epoxy matrix with lead circular inclusion of radius $R_{f}=0.35 \ell$ and rubber annular coating of external radius $R_{c}=0.45 \ell$.

Figure 11a shows, on the left, the effective surface density of the plate as a function of the frequency, normalized with respect the first eigenfrequency of the coating which, for this particular example, reads

$$
\omega_{1}=18.854 \sqrt{\frac{D_{c}}{\gamma_{c} R_{c}^{4}}} .
$$

The gap/mid-gap ratio of the first predicted bandgap is of $100 \%$, significantly higher than that obtainable with binary plates (cf. Figure 9 and Fig. 10). In the same figure, on the right, the dispersion curves obtained by Bloch-Floquet analysis confirm the prediction of the first two band gaps as intervals of negative effective mass and show the presence of several orthogonal modes of the coating inside them.

A further advantage of the analytical solution provided by (89), is the possibility to perform parametric studies on the band gaps without repeating many times the numerical Bloch-Floquet analyses. For instance, one can vary the radius $R_{f}$ of the inclusion, keeping fixed all the other quantities, and obtain the opening and closing frequency of the first three band gaps as a function of the ratio $R_{f} / R_{c}$. Figure $11 \mathrm{~b}$ shows the obtained band gaps, normalized with respect $\omega^{*}=\sqrt{D_{c} / \gamma_{c} R_{c}^{4}}$ : the width of the first band gap significantly increases with the radius of the inclusion.

\section{Conclusions}

In the present work, we employed the asymptotic homogenization technique to study the transversal vibrations at low frequencies of thin periodic plates. The homogenization is performed starting from the structural theory, and all elementary problems are formulated using 
the Kirchhoff theory, which is valid for small thickness. The method provides a general formula for the dynamic surface density of the plate, which depends only on its surface static density, the mass contrast between the two phases, and the geometry and the material stiffness of the inclusion. In particular, an analytical expression is obtained in the case of circular inclusions, while for square ones a numerical evaluation is performed.

The band gaps of the homogenized plate for in-plane propagating transversal waves correspond to the frequency intervals in which the effective surface density is negative. The same result is obtained considering the asymptotic homogenization of the Bloch-Floquet problem on the unit cell of the periodic plate, thereby demonstrating the equivalence of the two approaches.

Several examples allow us to compare the predicted band gaps with those obtained by numerical Bloch-Floquet analysis on the unit cell. When the stiffness contrast between the phases is very high (at least 2 to 3 orders of magnitude), the lower intervals of negative effective surface density correspond exactly to the band gaps of the real plate. The range of validity of the predictions increases as the stiffness contrast becomes larger. However, as discussed for the proposed two-phase plate, this affects significantly the amplitude of the band gaps.

Wider band gaps can be obtained in ternary plates. Therefore, we extended the homogenization approach to ternary plates with rigid inclusions coated by a soft material, and embedded in a stiff matrix. In the case of circular inclusions and annular coatings, we obtained an analytical expression of the effective surface density. A parametric study shows that the gap/midgap ratio of the first band gap increases as the radius of the inclusion increases.

The asymptotic analysis here developed allows to highlight peculiar features of metamaterial plates that are not exhibited by generic metamaterial solids and that can be exploited in practical applications. These features are: (i) the mechanism of local resonance, leading to band gap formation, can be obtained in plates constituted by a single material by changing periodically the thickness (purely geometric effect), and (ii) by reducing the ratio between the thickness and the in-plane dimensions of the inclusions one can lower the band gap frequency at fixed lattice dimensions.

Open Access This article is licensed under a Creative Commons Attribution 4.0 International License, which permits use, sharing, adaptation, distribution and reproduction in any medium or format, as long as you give appropriate credit to the original author(s) and the source, provide a link to the Creative Commons licence, and indicate if changes were made. The images or other third party material in this article are included in the article's Creative Commons licence, unless indicated otherwise in a credit line to the material. If material is not included in the article's Creative Commons licence and your intended use is not permitted by statutory regulation or exceeds the permitted use, you will need to obtain permission directly from the copyright holder. To view a copy of this licence, visit http://creativecommons.org/licenses/by/4.0/.

\section{References}

1. Allaire, G., Briane, M., Vanninathan, M.: A comparison between two-scale asymptotic expansions and Bloch wave expansions for the homogenization of periodic structures. SeMA J. 73(3), 237-259 (2016)

2. Asby, M.F.: Materials Selection in Mechanical Design, 4th edn. Elsevier, Amsterdam (2011)

3. Auriault, J.L., Bonnet, G.: Dynamique des composites élastiques périodiques. Arch. Mech. 37(4-5), 269-284 (1985). 1985

4. Auriault, J.L., Boutin, C.: Long wavelength inner-resonance cut-off frequencies in elastic composite materials. Int. J. Solids Struct. 49(23-24), 3269-3281 (2012)

5. Bakhvalov, N., Panasenko, G.: Homogenisation: Averaging Processes in Periodic Media. Kluwer Academic, Dordrecht (1989)

6. Bensoussan, A., Lions, J.L., Papanicolaou, G.: Asymptotic Analysis for Periodic Structures. NorthHolland, Amsterdam (1978) 
7. Bloch, F.: Über die Quantenmechanik der Elektronen in Kristallgittern. Z. Phys. 52(7-8), 555-600 (1929)

8. Caillerie, D.: Plaques élastiques minces à structure périodique de période et d'épaisseur comparables. C. R. Acad. Sci. Paris 294, 159-162 (1982)

9. Caillerie, D., Nedelec, J.C.: Thin elastic and periodic plates. Math. Methods Appl. Sci. 6(1), 159-191 (1984)

10. Comi, C., Driemeier, L.: Wave propagation in cellular locally resonant metamaterials. Lat. Am. J. Solids Struct. 15(4), 1-15 (2018)

11. Comi, C., Marigo, J.J.: Homogenization approach and Bloch-Floquet theory for band-gap prediction in 2D locally resonant metamaterials. J. Elast. 139(1), 61-90 (2020)

12. Comi, C., Moscatelli, M., Marigo, J.J.: Two scale homogenization in ternary locally resonant metamaterials. Mater. Phys. Mech. 44(1), 8-18 (2020)

13. Craster, R.V., Kaplunov, J., Pichugin, A.V.: High-frequency homogenization for periodic media. Proc. R. Soc. A, Math. Phys. Eng. Sci. 466(2120), 2341-2362 (2010)

14. D’Alessandro, L., Belloni, E., D’Alò, G., Daniel, L., Ardito, R., Corigliano, A., Braghin, F.: Modelling and experimental verification of a single phase three-dimensional lightweight locally resonant elastic metamaterial with complete low frequency bandgap. In: 11th Int. Congr. Eng. Mater. Platforms Nov. Wave Phenomena, Metamaterials 2017, pp. 70-77 (2017)

15. Dunford, N., Schwartz, J.T.: Linear Operators, Part II Spectral Theory. Self Adjoint Operators in Hilbert Space. Interscience Publishers (Wiley), New York, London, Sidney (1963)

16. Duvaut, G., Metellus, A.M.: Homogeneisation d'une plaque mince en flexion des structure periodique et symmetrique. C. R. Acad. Sci. A 283, 947-950 (1976)

17. Faraci, D., Comi, C.: Asymptotic homogenization of metamaterials elastic plates. J. Phys. Conf. Ser. 2015(012038), 1-5 (2021)

18. Floquet, M.G.: Sur les équations différentielles linéaires à coefficients périodiques. Ann. Sci. Éc. Norm. Supér. (2) 12, 47-88 (1883)

19. Hsu, J.C., Wu, T.T.: Lamb waves in binary locally resonant phononic plates with two-dimensional lattices Appl. Phys. Lett. 90(20) (2007) 201904

20. Kittel, C.: Introduction to Solid State Physics, 7th edn. Wiley, New York (1996)

21. Krushynska, A.O., Kouznetsova, V.G., Geers, M.G.D.: Towards optimal design of locally resonant acoustic metamaterials. J. Mech. Phys. Solids 71(1), 179-196 (2014)

22. Lewiński, T.: Effective models of composite periodic plates-I. Asymptotic solution. Int. J. Solids Struct. 27(9), 1173-1184 (1991)

23. Lewiński, T., Telega, J.J.: Plates, Laminates, and Shells: Asymptotic Analysis and Homogenization. World Scientific, Singapore (1999)

24. Liu, Z., Liu, Z., Zhang, X., Mao, Y., Zhu, Y.Y.: Locally resonant sonic materials. Science 289(5485), 1734-1736 (2000)

25. Phani, A.S., Woodhouse, J., Fleck, N.A.: Wave propagation in two-dimensional periodic lattices. J. Acoust. Soc. Am. 119(4), 1995-2005 (2006)

26. Wang, G., Wen, X., Wen, J., Shao, L., Liu, Y.: Two-dimensional locally resonant phononic crystals with binary structures. Phys. Rev. Lett. 93(15), 154302 (2004)

Publisher's Note Springer Nature remains neutral with regard to jurisdictional claims in published maps and institutional affiliations. 